\title{
Functional Outcomes of Surgical Treatment of the Fifth Diaphyseal Metatarsal Fractures (Dancer's Fractures). A Case-Series and a Literature Review
}

\author{
Grigorios Kastanis ${ }^{1 *}$, Kapsetakis $\mathbf{P}^{1}$, Magarakis $\mathbf{G}^{1}$, Stavrakakis $\mathbf{M I}^{1}$ \\ and Pantouvaki $\mathrm{A}^{2}$ \\ ${ }^{1}$ Department of Orthopaedic, General Hospital of Heraklion-Venizeleio, Crete, \\ Greece \\ ${ }^{2}$ Department of Physiotherapy, General Hospital of Heraklion-Venizeleio, Crete, \\ Greece
}

*Corresponding Author: Grigorios Kastanis, Department of Orthopaedic, General Hospital of Heraklion-Venizeleio, Crete, Greece.

Received: July 26, 2021

Published: August 04, 2021

(C) All rights are reserved by Grigorios

Kastanis., et al.

\begin{abstract}
Introduction: Fifth metatarsal fractures represent a significant proportion of injuries, while fractures of the distal diaphyses (dancer fractures) comprise $20 \%$ of all fifth metatarsal fractures. Conservative treatment is the preferred method for managing these fractures. The aim of this study is to present our functional outcomes of patients, in long term (one year), who underwent surgical treatment with low profile plates and to analyze the time of fracture union, the complications and final patient satisfaction.

Material and Method: A retrospective study was performed from January 2015 to November 2019 with forty one patients undergone surgical treatment with low profile locking plates $2,3 \mathrm{~mm}$ or screws for an unstable (spiral or oblique) fracture of distal diaphyses of $5^{\text {th }}$ metatarsal. The data collected, consisted of patient demographics, radiographic healing times, fracture characteristics, complications and final patients' satisfaction (AOFAS Ankle-Hindfoot scale). Fractures were classified into two types according to the anatomical location: Type I which is a long oblique fracture that begins distal to lateral surface of metatarsal neck and extends into diaphysis (23 cases 56,1\%), and type II which is when the fracture line starts at the distal-lateral metaphysis and extends proximal as spiral pattern (18 cases $43,9 \%)$.

Results: Twelve men and twenty nine females were assessed post-surgically with a mean follow-up of 16,2 months. Time to union in all fractures was $7,2 \pm 2,9$ weeks more specifically in type I the union $(6,1 \pm 1,1)$ was more quick than type II $(7,9 \pm 1,3)$. Complications encountered were in three cases a delay union, in four cases infection and one with malunion. At final examination the AOFAS Ankle -Hindfoot scale was for the type I, 91,5 (range 89 - 93) and for the type II, 89,7 (range 85 - 91). None of the patients presented at last examination with metatarsalgia and the implants were not removed in any patient.

Conclusion: Based on our results we postulate that open reduction and internal fixation of Dancer fractures offer high incidence of union, low rate of complications and should be considered as the ideal management for patients who need rapid reintegration into their previous activities.
\end{abstract}

Keywords: Dancer's Fracture; Fifth Metatarsal; Outcomes; Surgical Treatment; Complications

Citation: Grigorios Kastanis., et al. "Functional Outcomes of Surgical Treatment of the Fifth Diaphyseal Metatarsal Fractures (Dancer's Fractures). A Case-Series and a Literature Review". Acta Scientific Orthopaedics 4.9 (2021): 09-15. 


\section{Introduction}

Fractures of metatarsal bones are the most usual injury of the foot which appears more often in athletes (ballet), military personnel and European population [1]. Fifth metatarsal fractures ascertain to be the most common metatarsal fractures diagnosed in emergency department with a higher percentage in female and in older age comparatively with men, but with exception in children younger than fifth years old [2,3]. The percentage of fifth metatarsal fractures accounts to more than $50 \%$ of all metatarsal fractures while the fracture of diaphyses of the bone appears to take part in $20 \%$ of all $5^{\text {th }}$ metatarsal fractures [4].

The mechanism of distal diaphyseal $5^{\text {th }}$ metatarsal fracture (dancer's fractures) has been delineated as strong ground reactive forces are tangent over the head and neck of metatarsal when the foot is a plantar-flexion movement, and while the metatarsal base remains stable (from ligamentous attachments) creating a triplanar rotational force which leads to spiral or oblique fracture [5]. The fifth metatarsal bone is the largest one in the forefoot and is critical for weight bearing in the final portions of the stance phase. The obliquity pattern of this fracture untreated or maltreated may lead to shortening and elevation of metatarsal head which results in increased weight bearing under the fourth metatarsal, plantar callosities, metatarsalgia and disability [6].

Buddecke., et al. (2010) reported that with exception of fifth base metatarsal fracture, little standardization is available for treatment of distal diaphyseal fracture. In literature ambiguous opinions have been reported among the indications (displacement, angulation, shortening) of treatment (conservative or surgical) of these spiral or oblique fractures. In general, conservative treatment is an accepted method because researchers suggest that these types of fractures have large percentage of healing and satisfactory outcomes with either short leg cast or fracture shoe treatment [8]. Moreover existed opponents of the surgical therapy which suggest that when the displacement is more than $2 \mathrm{~mm}$ the patients present less favorable outcomes (metatarsalgia) and limitation of daily activity for months [1]. Shereff., et al. (1990) first classified these fractures according to the displacement in three types: nondisplaced, minimally displaced and displaced (more than $3-4 \mathrm{~mm}$ of separation or $10^{\circ}$ angulation) [7]. Recently Zwitser., et al. (2010) support that fractures of lesser metatarsal with displacement more than $3-4 \mathrm{~mm}$ or $>10^{\circ}$ angulation is suitable for open reduction and internal fixation [9].

\section{Aim of the Study}

The aim of this study is to present our functional outcomes of patients in long term (one year) who underwent surgical treatment with low profile plates-screws and to analyze the time of fracture union (radiographically), the complications (infection, delayed union, nonunion, metatarsalgia) and final patients satisfaction (AOFAS Ankle -Hindfoot scale).

\section{Materials and Methods}

This retrospective study analyzed 41 patients with a displaced fifth metatarsal diaphyseal fracture (dancer fracture) with a mean age of 47 years old (range from 23 to 59 years) from January 2015 to November 2019. Inclusion criteria were: I age over 20 years old, II unstable distal $5^{\text {th }}$ metatarsal diaphyseal fracture, III involvement of other diaphyseal metatarsal fractures, IV polytrauma. Exclusion criteria were: I age younger than 18 years old, II patients who received any surgical treatment from another hospital, III patients that were initially treated conservatively, IV minimum follow-up one year. The Institutional Ethical Committee approved this study.

From 41 patients, twelve were male and twenty-nine females. The cause of injury was: fall in 20 cases, twist in 15 and traffic accident in 6 cases. The right foot was involved in 30 cases and the left in 11 patients. In 5 cases there was an involvement of the diaphyses of the $4^{\text {th }}$ metatarsal. From the medical records of patients was found that fourteen cases were with Diabetes Mellitus (4 patients had type I and 10 had Type II) while in smoking status were 29 patients (Table 1). The average Body Mass Index (BMI) was 29.4 (range from 15.3 to 36,7 ). In all patients radiographic examination (AP and oblique views) was performed at the emergency department and in 5 cases (with concomitant $4^{\text {th }}$ metatarsal fracture) a ct/scan was done also, to exclude other injuries to the involved foot. Fractures were classified in two types according to the anatomical location as described by Thompson., et al. (2017): Type I is a long oblique fracture that begins distal to lateral surface of metatarsal neck and extending into diaphysis (23 cases $56,1 \%$ ) and type II when the fracture line starts at the distal-lateral metaphysis and extends proximal as spiral pattern (18 cases 43,9\%) [6]. As indication for surgical management we followed the below criteria: displacement more than $3 \mathrm{~mm}$ (range from 3 to 8,5 ), angulation $>8^{\circ}$ (range from 5 to 10 degree). 


\begin{tabular}{|c|c|c|}
\hline \multicolumn{2}{|c|}{ Data } & n (\%) \\
\hline \multirow[b]{2}{*}{ Gender } & Male & $12(29,3 \%)$ \\
\hline & Female & $29(70,7 \%)$ \\
\hline \multirow[b]{2}{*}{ Foot } & Right & $30(73,1 \%)$ \\
\hline & Left & $11(26,9 \%)$ \\
\hline \multirow[b]{2}{*}{ Diabetes } & Yes & $14(34,1 \%)$ \\
\hline & No & $27(65,9 \%)$ \\
\hline \multirow[b]{2}{*}{ Smoking } & Yes & $29(70,8 \%)$ \\
\hline & No & $12(29,2 \%)$ \\
\hline \multirow[b]{2}{*}{ MTT Involved } & Isolated $5^{\text {th }}$ & $36(87,8 \%)$ \\
\hline & $4^{\text {th }}$ and $5^{\text {th }}$ & $5(12,2 \%)$ \\
\hline \multirow{3}{*}{ Cause } & Fall & $20(48,8 \%)$ \\
\hline & Twist & $15(36,6 \%$ \\
\hline & Traffic accidence & $6(4,6 \%)$ \\
\hline
\end{tabular}

Table 1: Patients demographics.

The mean time to surgery was 3,4 days (varied from 2 - 7 days) depended on the good quality of soft tissues. All patients were operated with regional or general anesthesia (depended ASA score), in supine position with arm tourniquet and fluoroscopic control and all operations were performed by author. Dorsolateral approach was performed in all cases and after elevation of soft tissue we expose the fracture. After a meticulous irrigation the fracture was reduced with distraction and rotation while temporary stabilizations performed with reduction clump while the quality of reduction was controlled by fluoroscopy. In eighteen cases with type I the fracture was fixated with low profile locking plates $2,3 \mathrm{~mm}$ which performed in lateral surface of the $5^{\text {th }}$ metatarsal while in other 5 cases the fracture fixed with three cortical screws 2,3 mm (Figure 2). In cases with type II fractures in fourteen patients we applied plate while in rest cortical screws (Figure 1). A final control with C-arm intensifier was performed to check the final osteosynthesis (position of the plate, reduction of the fracture and the route of screws) and clinical fracture stability. The skin was closed in layers, and a half plaster of Paris cast was applied.

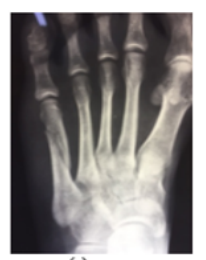

(a)
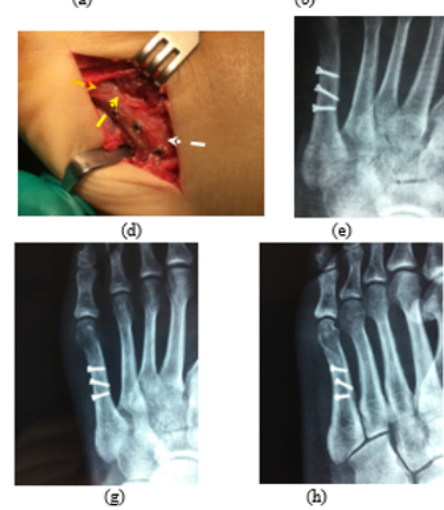

Figure 1: A Female with left type II diaphyseal $5^{\text {th }}$ metatarsal fracture, preoperative x-rays AP (a) and Oblique (b) views. Intraoperative long oblique fracture (c) yellow arrow fracture line, orange arrow head of $5^{\text {th }}$ metatarsal, white arrow base of metatarsal) and osteosynthesis with three screws (d) and postoperative x-rays AP (e) and Oblique (f). At one year postoperative $\mathrm{x}$-rays AP (g) and Oblique (h).

Postoperatively, all patients underwent a standard rehabilitation protocol program from the first postoperative day including walking with non-weight bearing with a cast for two weeks and with a functional ankle brace until radiographic evidence of union afterwards. Passive motion of the finger digits initiated immediately and patient was trained to perform active strengthening exercises of adjacent joints of lower limb.

\section{Results}

The mean follow-up is about 16,2 months (range from 13 months to 28 months). None of patients missed the last re-examination. Results were evaluated according to fracture union, determined by follow-up x-Rays, complications, AOFAS Ankle -Hindfoot scale. All cases were assessed in two weeks and in 1,3,6,12 months post surgically. Radiographic examination with 2 views 


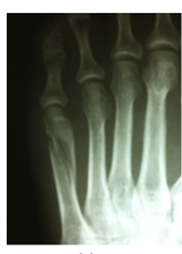

(a)

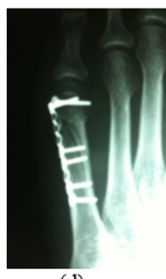

(d)

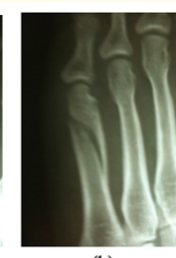

(b)

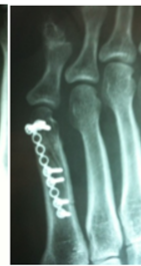

(e)

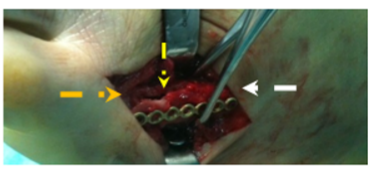

(c)

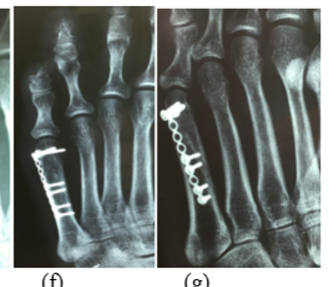

(g)
Figure 2: Female 42 years old with a right type I diaphyseal $5^{\text {th }}$ metatarsal fracture, preoperative x-Rays AP (a) and Oblique (b) views. Intraoperative long oblique fracture (c) yellow arrow fracture line with low profile plate, orange arrow head of $5^{\text {th }}$ metatarsal, white arrow base of metatarsal). Postoperative $\mathrm{X}$-rays AP (d) and Oblique (e). At one year postoperative x-rays AP (f) and Oblique (g).

(AP, Oblique) was performed in all cases postoperatively during follow-up until the evidence of union. Radiographic union was defined by complete bridging with callus the two ends of fracture and absence of fracture line, while clinical union determinated as absence of pain during walking and without assistance aids. Time to union in all fractures was 7,2 $\pm 2,9$ weeks, more specifically in type I the union $(6,1 \pm 1,1)$ was quicker than type II $(7,9 \pm 1,3)$ (Table 2). Complications encountered were three cases with delay union, four cases with infection and one with malunion. Infection was present in four cases (two type I and two in type II) in which after administration of antibiotics (cephalosporin) oral therapy the symptoms were disappeared. All these patients suffered from Diabetes Mellitus, and one patient was a smoker (25 cigarettes per day) and infection did not affect fracture union time.

Delay union appears in one patient of type I and in two of type II fractures. In cases with type I fractures the union achieved in 10 weeks respectively, while the other two cases (type II) in 12 and 13

\begin{tabular}{|l|c|c|c|}
\hline \multicolumn{2}{|c|}{ Variable } & Type I (23) & Type II (18) \\
\hline Age & $39(23-58)$ & $45(30-59)$ \\
\hline Gender: Male/Female & $8 / 15$ & $4 / 14$ \\
\hline Union Time (weeks) & $6,1 \pm 1,1$ & $7,9 \pm 1,3$ \\
\hline \multirow{4}{*}{ Complication } & Infection & 2 & 2 \\
\cline { 2 - 4 } & Delay union & 1 & 2 \\
\cline { 2 - 4 } & Nonunion & 0 & 0 \\
\cline { 2 - 4 } & Malunion & 0 & 1 \\
\hline \multirow{2}{*}{ AOFAS } & & \\
Ankle-Hindfoot Scale (1year) & $91,5(89-93)$ & $89,7(85-91)$ \\
\hline
\end{tabular}

Table 2: Fracture characteristics of type I and II.

weeks. All of them were smokers and two suffered from diabetes mellitus (type II). Among gender two were female and one male. The internal fixation in these cases included low profile locking plates in two cases and cortical screws in one only. The case with malunion was a 59 year-old female with a type II fracture without comorbidities but with a BMI 36. The paradox was that patient returned to previous daily activities without subfourth metatarsalgia and refuse reoperation. At final examination, the AOFAS Ankle -Hindfoot scale was for the type I 91, 5 (89 - 93) and for the type II 89, 7 (85 - 91). None of the patients presented at last examination with metatarsalgia and the implants were not removed in any patient.

\section{Discussion}

Fractures of the $5^{\text {th }}$ metatarsal are classified according to the anatomical region of the bone that appears so they are divided in head and neck fractures, shaft and fractures at the bone base. Vogler., et al. (1995) report that fracture of neck and shaft of $5^{\text {th }}$ metatarsal are typical spiral, in some cases with an associated butterfly fragment and significant comminution and important shortening [12]. The term "Dancer fracture" concerns an extrarticular displacement spiral or oblique fracture of distal diaphyses of $5^{\text {th }}$ metatarsal in which the distal bone fragment (head and neck) is elevated dorsally. Initially, Clapper., et al. (1995) delineated that the incidence of this type of fracture is $5 \%$ from all fractures of $5^{\text {th }}$ metatarsal, while O'Malley., et al. (1995) suggest that this fracture pattern is more common than once previously estimated $[10,11]$. 
The most common mechanism of this specific injury is twist or fall and blunt trauma and from literature appears in women's after the age of 55 years old, while Goulard., et al. (2008) report that dancer rolls over the outer border of the foot while in demipointe position on the ball of the foot, with the ankle fully plantar flexed is the most common cause of injury in ballet dancers [2,20]. Kane., et al. (2015) in a retrospective study of $12755^{\text {th }}$ metatarsals, report that mechanism of injury is a predictor for fracture location while gender and age have a role in fracture incidence [13]. Hasselman., et al. (2003) report that the $5^{\text {th }}$ metatarsal fracture has an incidence of $56,9 \%$ in women over 65 years old and that is an osteoporotic fracture [14]. In our study the most common cause of injury was fall $(48,8 \%)$ followed by twist injury $(36,6 \%)$. The majority of the cases were female $58,5 \%$ while in only $14(58,3 \%)$ female patients the age was over 50 years old ( 8 fractures type I and 4 type II).

The characteristic obliquity patterns of this fracture described by Konkel., et al. (2005) lead to a displacement and dorsal angulation of the proximal fractured segment [16]. The degree of displacement depends on the energy of the causal twisting injury, landing, or axial loading of the foot in the supinated position [20]. The major question posed was, what constitutes significant displacement and angulation? Shereff firstly suggests some guidelines among the displacement in three types but according to O'Malley they didn't support it from clinical or biomechanical reports $[7,11]$. Soave., et al. (2016) proposed a radiographic classification of distal shaft fracture of the fifth metatarsal with scope to assistance surgeon to treat this type of fracture [15]. The classification bases to the configuration of the fractures and amount of displacement and distingue the fracture in four grades. Recently Thompson., et al. (2017) described two types of fractures based on anatomic location of the fracture [6]. In our study we follow the last classification because we believe that describes mostly the fracture's morphology with great precision, assisting in the decision of treatment (conservative or surgical) and finally helps to preoperative planning to choose the implant.

The aim of treatment is to restore anatomic alignment of the fracture, to maintain a balanced metatarsal cascade with scope to attribute a pain free functional outcome [17]. Traditional conservative management of dancer fractures was the gold standard. O'Malley., et al. (1996) first report the results of conservative treatment with short leg weight bearing cast or an elastic wrap in 31 patients. The author applied this treatment in cases in which the displacement was less than $3 \mathrm{~mm}$ while in 4 cases with displacement more than $3 \mathrm{~mm}$ a closed or open reduction and internal fixation were applied [11]. Aynardi., et al. (2013) treated conservatively (casting and partial weight bearing) 141 cases with dancer fracture and average follow-up 3.5 years had only 2 cases with delay union and 3 cases with symptomatic nonunion. The researcher refers that initial displacement of the fracture was less than $5 \mathrm{~mm}$ and suggests strongly nonoperative management of this fractures [19]. Konkel., et al. (2005) in 16 cases present an average time to bony union 3,7 months and recommend nonoperative treatment of fifth metatarsal fractures for patients in whom the time to return to full activities is not critical [16]. It seems that the disadvantage of conservative treatment is the long time to union period when displacement or rotational deformity is remarkable, post injury metatarsalgia and prolonged time of immobilization and functional restriction.

Goulart., et al. (2008) suggested, in order to avoid prolonged immobilization and encourage faster return to sport applied surgical treatment in athletes dancer while Hardaker., et al. (1989) in fractures with remarkable misalignment applied intramedullary fixation with Kirschner wire [20,21]. The same point of view proposed by Boutefnouchet., et al. (2014), suggesting that dancer fracture with displacement more than $3-4 \mathrm{~mm}$, angular deformity more than $30^{\circ}$ and rotational deformity required closed or open reduction and stabilization with Kirschner wires or plates [22]. Recently, Thompson., et al. (2017) studying 64 cases with an average displacement of $3,20 \mathrm{~mm}$ and an angulation range of $5,9^{\circ}$, suggest surgical intervention, even for minimally displaced diaphyseal fractures, to maintain equal weight bearing across the metatarsal parabola [6]. In our cases the displacement was more than $3 \mathrm{~mm}$ (range from $3 \mathrm{~mm}$ to 8,5) and angulation more than $8^{\circ}$ (range from 5 to 10 degree). All patients underwent open reduction and internal fixation with low profile locking plates and screws 2,3 $\mathrm{mm}$, with a mean time to union 6,1 weeks for type I fractures and 7,9 weeks for type II.

Among the surgical treatment in literature there is no clinical protocol of which implant is indicated in this pattern of fracture and there is no clinical studies to compare the implant applied (plates versus Kirschner wire) or eventual complication (malunion, hardware irritation or removal) after choosing the one or 
the other implant. Curtis., et al. (2015) compared intramedullary Kirschner wires (crossed or intramedullary) and 2,5 nonlocking plates and report that plate concluded that plate fixation was 11 and 15 times stronger in 3-point bending that K-wires and the osteosynthesis with plate is more stable means of fixation [23]. Stavlas., et al. (2010), in order to evaluate the role of reduction and internal fixation of Lisfranc Fracture dislocation present that percentage of major complications in a systematic review were skin problems $(3,6 \%)$, infection $(1,5 \%)$ and metal implant complications $16,1 \%$ [24]. Murphy (2006) report that painful neuroma at site of incision and nerve irritation form the implant are identified after dorsal and lateral approach [25]. Bryant., et al. (2018) report that from 75 fractures treated with plate fixation 30\% present some degree of hardware irritation but none of patients removed the implant and the incidence of nonunion it was zero [17]. On the contrary, in cases in which Kirschner wires were selected for fixation of fractures it was obligated to remove the implant [26]. In our study all the plates were applied in the lateral surface of the bone, none of patients report discomfort from the implant and there was no removal of the implants in all cases.

Bone union process has been reported to be affected by many intrinsic factors (primary and secondary). Macintyre., et al. (2000) report that hormonal status, nutritional status, strength, flexibility and age, are factors that act in healing process [27]. Murphy (2006) reports that delay union is more in lesser metatarsals injury, smoking, systemic illnesses and immune compromise [25]. The three cases of nonunion from our fractures were all smokers and two suffered from diabetes mellitus type II. Maybe this reason was responsible for the nonunion.

This study is presented with the following limitations. First, the small number of dancer fractures ( 41 fracture) and second the quite short follow-up period (16,2 months). Possibly a larger sample of patients leads to more reliable results over a longer follow-up time regarding complication, type and rate. Third, in our practice we still use low profile locking plates for metatarsal fixation and we don't have any comparative functional results from treatment with Kirschner wires according to union rate (nonunion or malunion).

\section{Conclusion}

Distal diaphyseal shaft fractures (dancer fractures) are a common injury whose treatment requires careful evaluation because it may lead to malunion or nonunion which results to increased weight bearing under the fourth metatarsal, plantar callosities, metatarsal pain and functional disability. The two major fracture criteria (displacement and rotational deformity) should be the main guide for choosing treatment, conservative or surgical. Low profile plates offer a rigid fixation and stability of the fracture with low percentage of nonunion or malunion and should be considered as the ideal management for patients who need rapid reintegration to their previous activities.

\section{Conflict of Interest}

The authors declare that have no conflict of interest.

\section{Ethical Approval}

Our institution does not require ethical approval for reporting individual cases or case series.

\section{Consent}

Verbal informed consent was obtained from the patients for their anonymized information to be published in this article.

\section{Bibliography}

1. Cakir H., et al. "Demographics and outcome of metatarsal fractures". Archives of Orthopaedic and Trauma Surgery 131.2 (2011): 241-245.

2. Petrisor BA., et al. "The epidemiology of metatarsal fractures". Foot and Ankle International: SAGE Journals 27.3 (2006): 172-174.

3. Singer G., et al. "A study of metatarsal fractures in children". Journal of Bone and Joint Surgery American 90.4 (2008): 772776.

4. Buddecke DE., et al. "Metatarsal fractures". Clinics in Podiatric Medicine and Surgery 27.4 (2010): 601-624.

5. O'Shea MK., et al. "Clinical perspective of the treatment of fifth metatarsal fractures". Journal of the American Podiatric Medical Association 85.9 (1995): 473-480.

6. Thompson P., et al. "Surgical management of fifth metatarsal diaphyseal fractures: A retrospective outcomes study". The Journal of Foot and Ankle Surgery 56.3 (2017): 463-467. 
7. Shereff MJ. "Complex fractures of the metatarsals". Orthopedics 13.8 (1990): 875-882.

8. Gösele A., et al. "Early functional treatment of a 5th metatarsal fracture using an orthopedic boot". Swiss Surgery 3.2 (1997): 81-84.

9. Zwitser EW and Breederveld RS. "Fractures of the fifth metatarsal: diagnosis and treatment". Injury 41.6 (2010): 555-562.

10. Clapper MF., et al. "Fractures of the fifth metatarsal (analysis of a fracture registry)". Clinical Orthopaedics 315 (1995): 238-241.

11. O'Malley MJ., et al. "Fractures of the distal shaft of the fifth metatarsal. "Dancer's fracture". American Journal of Sports Medicine 24.2 (1996): 240-243.

12. Vogler H., et al. "Fifth metatarsal fractures: biomechanics, classification and treatment". Clinics in Podiatric Medicine and Surgery 12.4 (1995): 725-747.

13. Kane JM., et al. "The epidemiology of fifth metatarsal fracture". Foot and Ankle Specialist 8.5 (2015): 354-359.

14. Hasselman CT., et al. "Foot and ankle fractures in elderly white women Incidence and risk factors". Journal of Bone and Joint Surgery American 85.5 (2003): 820-824.

15. Soave RL., et al. "A new radiographic classification for distal shaft fifth metatarsal fractures". The Journal of Foot and Ankle Surgery 55.4 (2016): 803-807.

16. Konkel KF., et al. "Nonoperative treatment of fifth metatarsal fractures in an orthopaedic suburban private multi speciality practice". Foot and Ankle International: SAGE Journals 26.9 (2005): 704-707.

17. Bryant T., et al. "Union rate and rate of hardware removal following plate fixation of metatarsal shaft and neck fractures". Foot and Ankle International: SAGE Journals 39.3 (2008): 326-331.

18. Prisk VR., et al. "Forefoot injuries in dancers". Clinics in Sports Medicine 27.2 (2008): 305-320.

19. Aynardi M., et al. "Outcomes of nonoperative management of displaced oblique spiral fractures of the fifth metatarsal shaft". Foot and Ankle International: SAGE Journals 34.12 (2013): 1619-1623.

20. Goulart M., et al. "Foot and ankle fractures in dancers". Clinics in Sports Medicine 27.2 (2008): 295-304.

21. Hardaker WT Jr. "Foot and ankle injuries in classical ballet dancers". Orthopedic Clinics of North America 20.4 (1989): 621-627.

22. Boutefnouchet T., et al. "Metatarsal fractures: A review and current concepts". Trauma 160.3 (2014): 147-163.

23. Curtis BD., et al. "Fixation of metacarpal shaft fractures: biomechanical comparison of intramedullary nail crossed K-wires and plate-screw constructs". Orthopaedic Surgery 7.3 (2015): 256-260.

24. Stavlas P., et al. "The role of reduction and internal fixation of Lisfranc fracture dislocations: a systematic review of the literature". International Orthopaedics 34.8 (2010): 1083-1091.

25. Murphy GA. "Operative treatment of stress fractures of the metatarsals". Operative Techniques in Sports Medicine 14 (2006): 239-247.

26. Sánchez Alepuz E., et al. "Fractures of the central metatarsal". Foot and Ankle International: SAGE Journals 17.4 (1996): 200-203.

27. Macintyre J and Joy E. "Foot and ankle injuries in dance". Clinics in Sports Medicine 19.2 (2000): 351-368.

\section{Volume 4 Issue 9 September 2021 \\ (C) All rights are reserved by Grigorios Kastanis., et al.}

